\title{
Investigation of Infrared Image Prediction for Subsonic Exhaust Plume
}

\author{
Fei Mei ${ }^{\mathrm{a}}$, Shiguo Chen ${ }^{\mathrm{a}}$, YingHong Li ${ }^{\mathrm{a}}$, Yong Jiang ${ }^{\mathrm{a}}$, Jing Cai ${ }^{\mathrm{b}}$, ShuKun ${ }^{\mathrm{b}}$, Zhang $^{\mathrm{b}}$ \\ ${ }^{a}$ College of Engineering Air Force Engineering University Xi'an, Shannxi Province, China \\ ${ }^{\mathrm{b}}$ Changcheng Institute of metrology \& Measurement Beijing, China
}

\begin{abstract}
An infrared imaging prediction model of exhaust plume was developed to understanding the infrared characteristics of exhaust plume. The method is based on the irradiance calculation of all pixels on the focal plane array. In order to compute the irradiance incident on each pixel, the gas radiation transfer path in the plume for the instantaneous field of view (IFOV) corresponds to the pixel was solved by the simultaneous equation of a cylinder which covers the exhaust plume and the line of sight. Radiance for the transfer path was calculated by equation of radiation transfer for nonscattering gas. The radiative properties of combustion were computed by Malkmus model with EM2C narrow band database $(25 \mathrm{~cm}-1)$. The pressure, species concentration for the path was determination by CFD analysis. The relatively intensity of each pixel was transferred to color in the display according to gray map coding and hot map coding. Infrared image of the exhaust plumes from a subsonic axisymmetric nozzle was predicted with the model. By changing the parameters, such as FOV and space resolution, the image of different imaging system can be predicted for varying relatively position of camera and the plume.
\end{abstract}

Index Terms: Exhaust plume; Infrared imaging; Radiative transfer equation; colormap

(C) 2012 Published by MECS Publisher. Selection and/or peer review under responsibility of the Research Association of Modern Education and Computer Science.

\section{Introduction}

The Infrared guided missiles have emerged as one of the most deadly threats to aircraft, they have been responsible for most of the military and civilian aircraft downed in the last several decades[1]. They can acquire and intercept aircraft by passively detecting IR-radiation (heat signatures) from them. Thus, they have truly fire and forget capability[2]. Recently, IR guided missile has advanced from un-cooled reticle seeker to multi-color array imaging seeker. To understand and counter the threat of these heat seeking missiles it is necessary to understand the characteristic of target's IR Imaging.

* Corresponding author.

E-mail address: meifeifff@163.com, Shukun_9869@qq.com 
The typical radiation sources of jet aircraft are the hot-metal tail-pipe, the exhaust gas plume, metallic skin[3]. Skins of fuselage and tailpipe are usually considered as gray body, whose Infrared image can simulate by its emissivity and temperature. As the emissivity of gases changes sharply with wavelength for a mixture of $\mathrm{H}_{2} \mathrm{O}, \mathrm{CO}_{2}, \mathrm{CO}$ and $\mathrm{O}_{2}$, It is not true for exhaust plume. The gas thickness, temperature field and gas composition all affected the radiation along given path.

This paper concentrated on infrared imaging simulation for exhaust plume with the intent to give additional information for modeling and understanding of the infrared characteristics of exhaust plume. This knowledge is necessary both in evaluating the threat against civilian aircraft and designing aircraft less susceptible to heat seeking missiles.

\section{Brief Description of Infrared Imaging}

\section{A. Infrared Imaging System}

There are two families of infrared imaging system: one is scanning system which creating a simulated image of the source by moving the IR signal over the detector. This is achieved by rotating a couple of offset mirrors or risley prisms to produce a specified pattern on the detector; One is staring systems are characterized by a two dimensional array of detector pixels which are scanned electronically and the image of the target was produced on the focal plane (see fig 1).

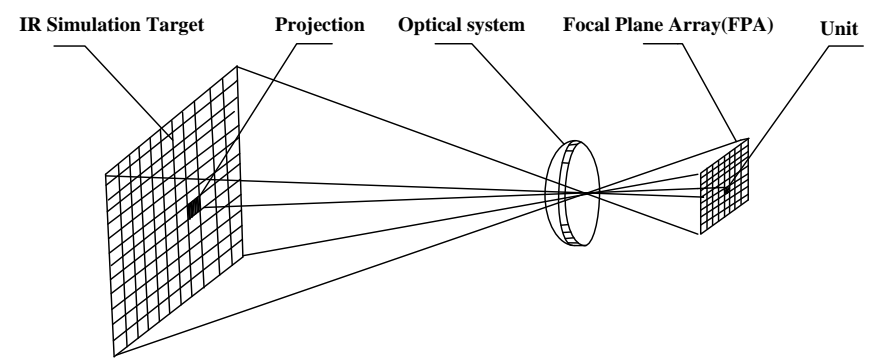

Fig.1 Schematic illustration showing of IR imaging

One pixel of the staring focal plane array receives radiation from its own IFOV. The pixel converted the radiation to electrical signal according to its irradiance, and the relatively intensity was transferred to color in the display according to some color map. Infrared image in the display was constituted by a group of pixels. The pixels were allocated as form as $128 \times 128,320 \times 240$ which is called detector spatial resolution. Color of each pixel is determined by the amount of irradiance on corresponding FPA unit.

\section{B. Irradiance on the FPA}

The pixel of the FPA has a size of $a \times b$, focus of the optical system is $f$, distance between the source and the lens is $R$, the projection size of the IFOV on the source is $a^{\prime} \times b^{\prime}$.It is obviously that:

$$
a \times b / f^{2}=a^{\prime} \times b^{\prime} / R^{2}
$$

The radiance of source in the corresponding IFOV is $L$.The projected area is $A$.The solid angle is $\Omega$ which is determined by the distance $R$ and diameter $D_{0}$ of the Len. The transmittance of optic system and atmospheric is $\tau_{0}$ and $\tau(R)$ respectively. The radiant flux of one pixel received from the source is 


$$
\begin{aligned}
\Phi=L A \Omega \tau_{0} \tau(R) & =L a^{\prime} b^{\prime} \pi D_{0}^{2} / 4 R^{2} \\
& =L a b \pi D_{0}^{2} \tau_{0} \tau(R) / 4 f^{2}
\end{aligned}
$$

The irradiance incident on the pixel is

$$
E=\Phi / a b=L \pi D_{0}^{2} \tau_{0} \tau(R) / 4 f^{2}
$$

The equation (3) implied that the irradiance of specific pixel was only relied on the radiance $L$ of plume which in the corresponding IFOV.

\section{Gas Radiation Modelling}

When the object was metal, the radiance in the IFOV can be calculated by Planck's law with the emissivity. Unfortunately, that's not true for exhaust plume. Because radiation emitted by gas is at discrete frequency, a characteristic of the vibrational mode. The number, width, and emissive powers of the various bands, depend on the gas composition, pressure, temperature, and thickness of gas volume. The variation of the spectral radiation intensity along a path is described by the RTE, which is an integral-differential equation that describes the spectral radiation intensity along a path in a fixed direction through an absorbing, emitting, scattering medium.

\section{A. Equation of Radiation Transfer for Nonscattering Gas}

In the RTE, the attenuation of intensity due to the absorption and scattering, and the augmentation of intensity resulted from the emission and scattering to the path are included.

Since it has few scattering media, the RTE in nonscattering media was exactly right for aircraft exhaust plume. When the distributions of pressure, temperature, and species concentration are given explicitly as functions of the path distance $\mathrm{u}$, the equation of transfer reads

$$
L_{\omega}(\omega, u)=\int_{0}^{u} L_{\omega}^{u}\left(\omega, u^{\prime}\right) \frac{\partial}{\partial u^{\prime}} \tau\left(\omega, u^{\prime}, u\right) d u^{\prime}
$$

For a given path which was divided into a number of isothermal columns, the equation is usually transformed into a numerical summation form

$$
L_{\omega}=-\sum_{m=1}^{m=N} L_{\omega}\left(T_{m}\right)\left(\tau_{\omega, m}-\tau_{\omega, m-1}\right)
$$

Where the transmittance is

$$
\tau_{\omega, m}=\exp \left(-\int_{0}^{u_{m}} \kappa_{\omega} d u\right)
$$

To obtain the radiance for a waveband, the spectral radiance should be integrated across frequency,

$$
L=\int_{\omega_{1}}^{\omega_{2}} L_{\omega} d \omega
$$

As absorption coefficient $\kappa_{\omega}$ is usually a very rapidly varying function of wavenumber, A precise determination of $L_{\text {requires evaluation of (6) at a very large number of frequencies. Such detailed calculations }}$ may be impractical or undesirable. The gas absorption coefficient varies much more rapidly across the spectrum than other quantities, such as blackbody intensity, etc. It is, therefore, in principle possible to replace the actual absorption coefficient (and intensity) by smoothened values appropriately averaged over a narrow spectral range.

$$
L_{b \omega}=-\sum_{m=1}^{m=N} L_{b \omega}\left(T_{m}\right)\left(\tau_{b \omega, m}-\tau_{b \omega, m-1}\right)
$$


$\tau_{b \omega, m}$ is a smoothened transmittance over a narrow spectral range defined by the band model.

\section{B. Band Model}

Band models are hypothetical models of simplified mathematical structure which are introduced to provide fair representations of the properties of real spectra at reasonable computing cost[4]. In general, a model consists of a set of lines in a spectral interval with specified properties regarding the intensities, shape, number, and distribution of the lines. Band model consists of Wide Band Model (WBD) and Narrow Band Model (NBD).NBD average the absorption coefficient over a smaller spectral interval, with higher accuracy than WBD.

A number of such "narrow band models" have been developed some 40-50 years ago. They were divided into regular band model (Elsasser Model) and statistical band model according to their own assumptive spectral lines distribution.

We can find several statistical band models which all assume that the positions of the individual spectral lines occur at random and that all lines have identical shape, differing only in strength. Such as Lines of equal strength, exponential line strength distribution (Goody Model), exponential tailed line strength distribution (Malkmus Model)[5]. The radiative properties of combustion were computed by Malkmus model which having a good agreement with Line by Line method with EM2C narrow band database $(25 \mathrm{~cm}-1)$ [6] here.

$$
\bar{\tau}=\exp \left[-2 \frac{\gamma}{d}\left(\sqrt{1+x p l k \frac{d}{\gamma}}-1\right)\right]
$$

For Inhomogeneous gas columns, the Curtis-Godson approximation is convenient.

$$
\left\{\begin{array}{l}
k_{e q}=\sum x_{i} p_{i} l_{i} k_{i} / \sum x_{i} p_{i} l_{i} \\
\left(\frac{\gamma}{d}\right)_{e q}=\sum x_{i} p_{i} l_{i} k_{i}\left(\frac{\gamma_{i}}{d_{i}}\right) / \sum x_{i} p_{i} l_{i} k_{i}
\end{array}\right.
$$

\section{Description of Exhasut Plume Infrared Imaging Prediction}

\section{A. CFD Analysis}

An implicit, coupled, CFD solver with standard k- $\varepsilon$ turbulance model has been used to model the plume flowfield emnating from the nozzle.

\section{B. The Gas transfer Path for Pixel}

The camera is at $90^{\circ}$ aspect angle. For simplify a cylinder that enclosure the exhaust plume was considered as the source. To calculate the radiance in the pixel's IFOV, we find Gas transfer path for the pixel. The path is between intersection A and B which are the point of intersection for the cylinder and the line of sight. It can be determined by the position of the cylinder, distance and the focus the pixel position in the FPA.

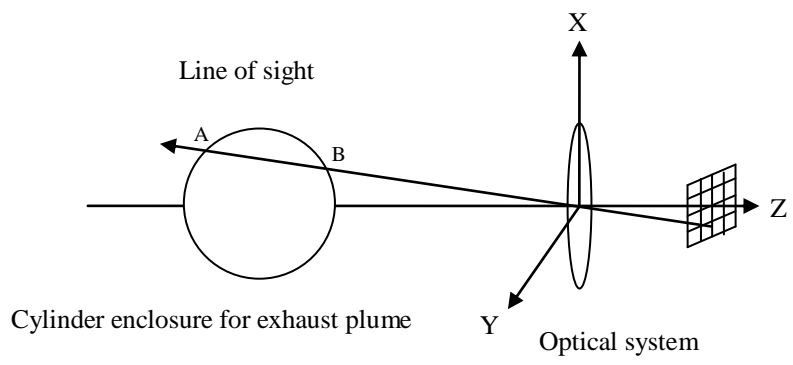

Fig. 2 Gas transfer path for pixel 


\section{Gas Radiance Calculation along the Transfer Path}

The path was divided into a number of isothermal columns. The pressure, temperature and concentration of water vapour, carbon dioxide, and carbon monoxide, the three gases which emit most strongly in the infrared along the path was provided by the CFD analysis. The detailed method to compute radiance for the given path has been discussed in section III.

\section{Atmospheric Transmission}

Radiant flux from the exhaust plume is selectively absorbed by several atmospheric gases and scattered away by small particles suspended in atmosphere (aerosols). The absorption and scattering are usually considered as the topic of extinction which causes attenuation in the amount of radiant flux passing through the atmosphere. The transmission of the atmosphere present in between the scene and the sensor is calculated by various numerical models that on the basis of the absorption, scattering and refractive-index fluctuations or turbulence, such as LOWTRAN[7], MODTRAN and FASCODE. These were developed by Air Force Geophysics Laboratory, USA. There are also some other models such as 4A/OP[8] initially developed at LMD (Laboratoire de Météorologie Dynamique).

LOWTRAN 7 is a low-resolution propagation model and computer code for predicting atmospheric transmittance and background radiance from 0 to $50,000 \mathrm{~cm}-1$ at a resolution of $20 \mathrm{~cm}-1$ which has been validated against field measurements. It is suitable for low altitudes(less than $40 \mathrm{~km}$ ) and at moderate temperatures.. The code is based on the LOWTRAN 6 [9] model. Multiple scattered radiation has

The LOWTRAN-7 code has been used in present analysis for computing atmospheric transmissivity.

\section{E. Color Mapping for Display}

The dynamic response range for an infrared camera is $E_{\min }^{0}$ to $E_{\max }^{0}$, The irradiance put on the detector's FPA varies from $E_{\min }$ to $E_{\max }$, and

$$
E_{\text {min }}^{0} \leq E_{\text {min }} \leq E_{\text {max }} \leq E_{\text {max }}^{0}
$$

The irradiance $E$ was normalized by

$$
I=\text { Round }\left(\frac{E-E_{\min }}{E_{\max }-E_{\min }} \times \text { color_number }\right)
$$

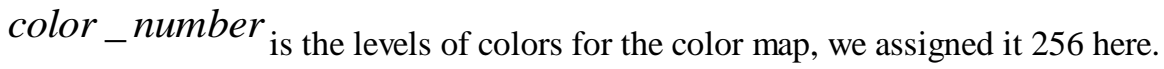

Two color maps were employed to render the pixels. The coding from $I$ to RGB are showed in fig3 and fig4 respectively.

1) Gray

Gray returns a linear grayscale colormap (Fig3).
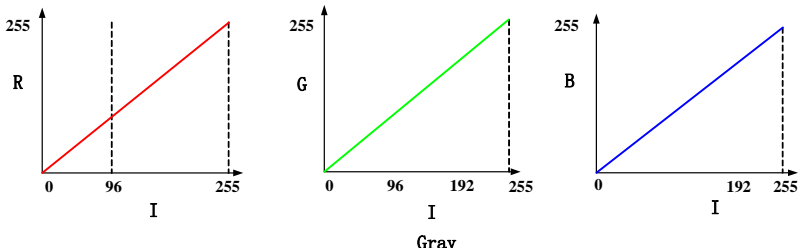

Fig. 3 Gray map coding

2)Hot

Hot varies smoothly from black through shades of red, orange, and yellow, to white (Fig4). 

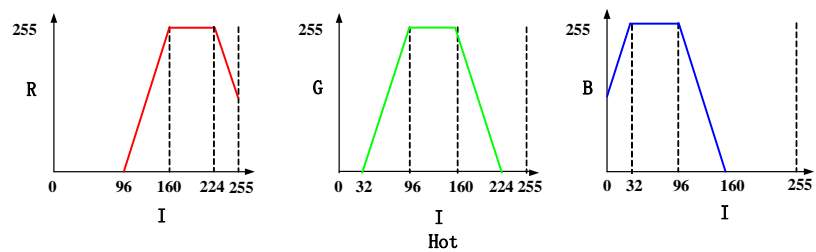

Fig. 4 Hot map coding

\section{Result and Conclusion}

The infrared imaging simulation was conducted for the exhaust plumes of a subsonic axisymmetric nozzle. The diameter of the nozzle exit is $20 \mathrm{~cm}$. The essential boundary parameters for CFD analysis are listed in table I.

\section{TABLE I BOUNDARY PARAMETERS FOR CFD}

\begin{tabular}{|c|c|c|}
\hline & Nozzle exit & environment \\
\hline Total pressure(pa) & 104510 & 96700 \\
\hline Static pressure(pa) & 96700 & 96700 \\
\hline Total temperature(K) & 590 & 296 \\
\hline Mole fraction of $\mathrm{CO}_{2}$ & 0.1 & $3.79 \times 10^{-4}$ \\
\hline Mole fraction of $\mathrm{H}_{2} \mathrm{O}$ & 0.1 & 0.005 \\
\hline
\end{tabular}

The total FOV of the infrared camera was supposed $22 \times 15^{\circ}$, image size is $320 \times 240$. The spectral interval for simulation is $2000-5000 \mathrm{~cm}-1$. The model can predict the infrared image of different imaging system by changing the parameters, such as FOV and space resolution.

The camera is placed at $90^{\circ}$ aspect angle, $500 \mathrm{~cm}$ away from the plume centerline and $220 \mathrm{~cm}$ away from the exit. The predicted infrared image is

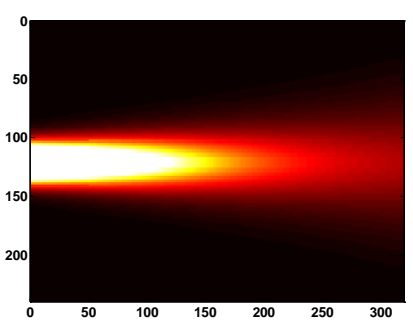

a) hot mapped image

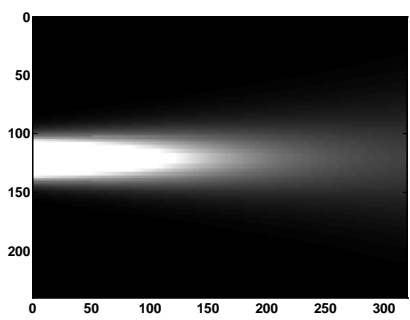

b) gray mapped image

Fig. 5 IR image for $500 \mathrm{~cm}$ away

Change the relatively position of camera and plume, the image can be predicted properly well too. Fig 4 show the predicted infrared image when the camera is placed at $90^{\circ}$ aspect angle, $400 \mathrm{~cm}$ away from the plume centerline and $200 \mathrm{~cm}$ away from the exit. 


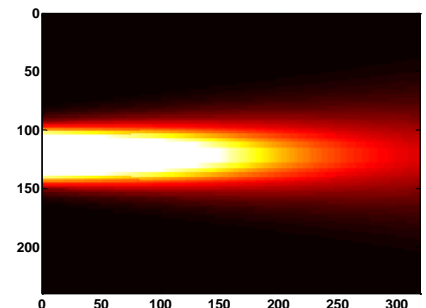

a) hot

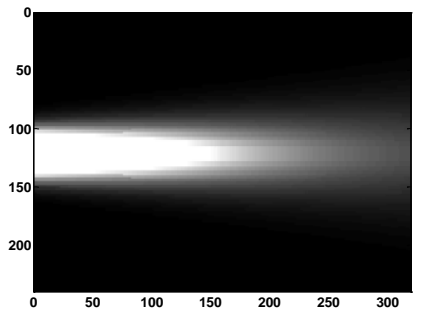

b) gray

Fig. 6 IR image for $400 \mathrm{~cm}$ away

The predicted image was showed reasonable, but it also need some experiment to validation which is planned to be done with an auxiliary power unit of a commercial airplane soon.

\section{Acknowledgment}

The authors are grateful to master candidate Bing Wen, Air Force Engineering University, for the systematic discussion of the Computation fluid dynamic knowledge. The authors also thank XuDong, Li of Xi'an Institute of Applied Optics for his many beneficial suggestions of the infrared imaging.

\section{References}

[1] Hughes D. and Wall R, "Missile Attack on DHL Jet Keeps Self-Defense Issue Bubbling," Aviation Week \& Space Technology November 2003.

[2] Understanding the infrared threat, Journal of Electronic Defense, vol.22 no.2, February 1999.

[3] S.P. Mahulikar, G.A. Rao, H.R. Sonawane, etc, “ Infrared signature studies of airborne target," Poceedings of the International Conference on Aerospace Science and Technology, India, 2008.

[4] C.B. Ludwing, W. Malkmus, J.E. Reardon, J.A.L. Thomson, "Handbook of Infrared Radiation from Combustion," NASA-SP-3080, 1973.

[5] Malkmus, W., "Random band Lorentz with exponential tailed $S^{-1}$ line-intensity distribution function," Journal of the Optical Society of America. vol.57, no.3, pp. 323-329, 1967.

[6] Saufiani, A. and Taine, J, "High temperature gas radiative property parameters of statistical narrow-band model for $\mathrm{H}_{2} \mathrm{O}, \mathrm{CO}_{2}$ and $\mathrm{CO}$, and correlated-K model for $\mathrm{H}_{2} \mathrm{O}$ and $\mathrm{CO}_{2}$," Int. J. of Heat Mass Transfer, vol. 40, no.4, pp. 987-991,1997

[7] Kneizys, F.X., Shettle, E.P.etc, “Lowtran 7 computer code: user's Manual,”AFGL-TR-88-0177, Hanscom AFB, MA 1988.

[8] L. Chaumat, C. Standfuss, B. Tournier, R. Armante and N. A. Scott, "4A/OP Reference Documentation," NOV-3049-NT-1178-v4.0, NOVELTIS, LMD/CNRS, CNES. 2009.

[9] Kneizys, F.X., Shettle, E.P.etc, "Atmospheric Transmittance /Radiance: Computer Code LOWTRAN 6," Air Force Geophysics Laboratory, Report AFGL-TR-83-0187, Hanscom AFB, MA. 1983. 